\title{
SOME CONVERGENCE THEOREMS FOR CONTINUED FRACTIONS ${ }^{1}$
}

ROBERT HELLER, JR.

1. Introduction. If each of $a$ and $b$ is a complex number sequence, then $F(a, b)$ denotes the sequence of approximants of the continued fraction

$$
\frac{1}{b_{1}}+\frac{a_{1}}{b_{2}}+\frac{a_{2}}{b_{3}}+\ldots
$$

i.e., $\quad F_{0}=0, \quad F_{1}=1 / b_{1}, \quad F_{2}=b_{2} /\left(b_{2} b_{1}+a_{1}\right), \cdots, \quad F_{p}=A_{p} / B_{p}, \cdots$, where $A_{0}=0, A_{1}=1, B_{0}=1, B_{1}=b_{1}, A_{p+1}=b_{p+1} A_{p}+a_{p} A_{p-1}$, and $B_{p+1}=b_{p+1} B_{p}+a_{p} B_{p-1} .{ }^{2}$ The notation $f(a)$ and $f_{0}, f_{1}, f_{2}, \cdots$ will be used for the sequence of approximants only if $b_{p}=1$. A necessary condition for convergence of $f(a)$ is that either $a_{n}=0$ for some positive integer $n$ or $a_{p} \neq 0$, and the series $\sum\left|c_{p}\right|$ diverges, where $c_{1}=1$ and $c_{p+1}=1 /\left(c_{p} a_{p}\right)$ (von Koch [4, pp. 27-29]). ${ }^{3}$ We will say that a sequence $a$ has property $V$, denoted " $a \in V$," only if this necessary condition is satisfied by the sequence $a$.

Scott and Wall [3] investigated continued fractions $f(a)$ such that for some non-negative term number sequence $r$,

$$
\begin{gathered}
r_{1}\left|1+a_{1}\right|>\left|a_{1}\right|, \quad r_{2}\left|1+a_{1}+a_{2}\right|>\left|a_{2}\right|, \text { and } \\
r_{p+2}\left|1+a_{p+1}+a_{p+2}\right| \geqq r_{p+2} r_{p}\left|a_{p+1}\right|+\left|a_{p+2}\right| .
\end{gathered}
$$

With certain restrictions on the $r_{p}$ (e.g., $r_{p}=1$ ), they showed that these inequalities imply that $f(a)$ converges only if $a \in V$. Lane and Wall [2] observed that the inequalities (1.1) (with no restriction on the $r_{p}$ other than $r_{p} \geqq 0$ ) imply the absolute convergence of the even and odd parts of $f(a)$ (i.e., the convergence of the series $\left|f_{0}\right|$ $+\sum\left|f_{2 p}-f_{2 p-2}\right|$ and $\left.\left|f_{1}\right|+\sum\left|f_{2 p+1}-f_{2 p-1}\right|\right)$ and then proved the following theorem.

Theorem LW. Any continued fraction $f(a)$ whose even and odd parts [4, pp. 20-21] are absolutely convergent converges only if $a \in V$. Thus,

Presented to the Society, November 29, 1958; received by the editors November 25, 1959.

1 This paper is based on the author's doctoral dissertation, written at The University of Texas, under the supervision of Professor H. S. Wall.

2 Throughout this paper, it will be understood that every statement involving the subscript $p$ is true for every positive integer $p$.

In this paper, numbers in brackets refer to the bibliography at the end of this paper. 
the condition that $a \in V$, which is NECESSARY for convergence of any continued fraction $f(a)$, is NECESSARY AND SUFFICIENT for convergence of those continued fractions $f(a)$ whose even and odd parts converge absolutely.

Theorem A of this paper states a necessary and sufficient condition (involving a certain set of inequalities) for absolute convergence of the even and odd parts of the continued fraction $F(a, b)$; these inequalities are, therefore, a generalization of the inequalities (1.1). In $\$ 3$ of this paper, the convergence of some particular continued fractions is proved by use of Theorem A.

"The Parabola Theorem" of Scott and Wall [3] may be regarded as a generalization of the theorem of Worpitzky $[4, \mathrm{p}$. 42] to the effect that $f(a)$ converges if $\left|a_{p}\right| \leqq 1 / 4$. Pringsheim [4, §11] showed that $f(a)$ converges if there exists a number sequence $g$ such that $0<g_{p}<1$ and $\left|a_{p}\right| \leqq\left(1-g_{p}\right) g_{p+1}$. If $0<r<1,0<g_{p}<1$, and $\left|a_{p}\right|$ $-\operatorname{Re} a_{p} \leqq 2 r\left(1-g_{p}\right) g_{p+1}$, then $f(a)$ converges only if $a \in V[4, \mathrm{pp}$. 142-143]. Wall [4, p. 143] offered the conjecture that this might also be true even if $r=1$. Lane $[1, \mathrm{p} .911]$ showed that if $r=1$ and, in addition to the above inequalities, there exists a positive number $M$ such that $\left|a_{p}\right|<M\left(1-g_{p}\right) g_{p+1}$, then $f(a)$ converges absolutely.

In $\$ 4$ of this paper, the aforementioned conjecture of Wall is settled in the negative and some additional conditions on the sequence $g$ are found which are necessary in order for the statement to be true.

2. A necessary and sufficient condition for absolute convergence of the even and odd parts of the continued fraction $F(a, b)$. The " $k$-inequalities" for the continued fraction $F(a, b)$ are:

(2.1) and

$$
\left|B_{2}\right|-k_{1}\left|a_{1} B_{0}\right| \geqq M_{0}\left|b_{2}\right|,\left|B_{3}\right|-k_{2}\left|a_{2} B_{1}\right| \geqq M_{1}\left|b_{3}\right|,
$$

$$
\begin{aligned}
\left|b_{p+1}\right|\left(\left|B_{p+3}\right|-k_{p+2} \mid\right. & \left.a_{p+2} B_{p+1} \mid\right) \\
& \geqq \frac{\left|b_{p+3} a_{p+1}\right|}{k_{p}}\left(\left|B_{p+1}\right|-k_{p}\left|a_{p} B_{p-1}\right|\right),
\end{aligned}
$$

where $k$ is a positive term number sequence and each of $M_{0}$ and $M_{1}$ is a positive number.

Note 2.1. If no term of the sequence $b$ is 0 and (2.1) holds, even with the first two inequalities weakened to $\left|B_{2}\right|-k_{1}\left|a_{1} B_{0}\right| \geqq 0$, $\left|B_{3}\right|-k_{2}\left|a_{2} B_{1}\right| \geqq 0$, then it can be shown by mathematical induction that $B_{p-1} \neq 0$. Thus, under these conditions, if some term of the sequence $a$ is 0 , then $F(a, b)$ converges absolutely. 
TheOREM A. Suppose that no term of the sequence $a$ is $0, j$ is one of the numbers 0 or 1 , and $b_{2 p-j} \neq 0$. The following two statements are equivalent:

(1) there exists a positive number $M_{j}$ and a positive term number sequence $\left\{k_{2 p-1+j}\right\}_{p=1}^{\infty}$ such that

and

$$
\frac{\left|B_{2+j}\right|-k_{1+j}\left|a_{1+j} B_{j}\right|}{\left|b_{2+j}\right|} \geqq M_{j}
$$

and

$$
\begin{aligned}
\frac{\left|B_{2 p+2+j}\right|-k_{2 p+1+j}\left|a_{2 p+1+j} B_{2 p+j}\right|}{\left|b_{2 p+2+j}\right|} \geqq \frac{\left|a_{2 p+j}\right|}{k_{2 p-1+j}} \\
\cdot \frac{\left|B_{2 p+j}\right|-k_{2 p-1+j}\left|a_{2 p-1+j} B_{2 p-2+j}\right|}{\left|b_{2 p+j}\right|},
\end{aligned}
$$

(2) the series $\left|F_{j}\right|+\sum_{p=0}^{\infty}\left|F_{2 p+2+j}-F_{2 p+j}\right|$ converges.

Proof. Suppose that statement (1) is true. Then, by mathematical induction, $\left|B_{2 p-2+j}\right|>0$. Now, if $a_{0}$ denotes 1 , then

$$
\begin{aligned}
\left|F_{2+j}-F_{j}\right| & =\frac{\left|a_{j} b_{2+j}\right|}{\left|B_{j} B_{2+j}\right|} \\
& \leqq \frac{\left|a_{j}\right|}{\left|B_{j}\right| M_{j}}\left(1-\frac{k_{1+j}\left|a_{1+j} B_{j}\right|}{\left|B_{2+j}\right|}\right)
\end{aligned}
$$

and

$$
\begin{aligned}
\left|F_{2 p+2+j}-F_{2 p+j}\right|= & \frac{\left|a_{1} a_{2} \cdots a_{2 p+j} b_{2 p+2+j}\right|}{\left|B_{2 p+j} B_{2 p+2+j}\right|} \\
= & \left|a_{j}\right| \frac{\left|a_{1+j} k_{1+j} a_{3+j} k_{3+j} \cdots a_{2 p-1+j} k_{2 p-1+j}\right|}{M_{j}\left|B_{2 p+j} B_{2 p+2+j}\right|} \\
& \cdot M_{j} \frac{\left|a_{2+j} a_{4+j} \cdots a_{2 p+j}\right|}{k_{1+j} k_{3+j} \cdots k_{2 p-1+j}}\left|b_{2 p+2+j}\right| .
\end{aligned}
$$

From the inequalities of statement (1),

$$
\begin{aligned}
M_{j} \frac{\left|a_{2+j} a_{4+j} \cdots a_{2 p+j}\right|}{k_{1+j} k_{3+j} \cdots k_{2 p-1+j}} \cdot\left|b_{2 p+2+j}\right| & \\
& \leqq\left|B_{2 p+2+j}\right|-k_{2 p+1+j}\left|a_{2 p+1+j} B_{2 p+j}\right| .
\end{aligned}
$$

Consequently, 


$$
\begin{aligned}
& \begin{aligned}
\left|F_{2 p+2+j}-F_{2 p+j}\right| \leqq \frac{\left|a_{j}\right|}{M_{j}}[ & \frac{\left|a_{1+j} k_{1+j} a_{3+j} k_{3+j} \cdots a_{2 p-1+j} k_{2 p-1+j}\right|}{\left|B_{2 p+j}\right|} \\
& \left.-\frac{\left|a_{1+j} k_{1+j} a_{3+j} k_{3+j} \cdots a_{2 p+1+j} k_{2 p+1+j}\right|}{\left|B_{2 p+2+j}\right|}\right]
\end{aligned} \\
& \text { from which }
\end{aligned}
$$

$$
\sum_{p=0}^{\infty}\left|F_{2 p+2+j}-F_{2 p+j}\right| \leqq \frac{\left|a_{j}\right|}{\left|B_{j}\right| M_{j}} .
$$

Suppose that statement (2) is true. Then $B_{2 p-2+j} \neq 0$ and

$$
\left|F_{2 p+2+j}-F_{2 p+j}\right|=\frac{\left|b_{2 p+2+j} a_{j} a_{1+j} \cdots a_{2 p+j}\right|}{\left|B_{2 p+j} B_{2 p+2+j}\right|}>0 .
$$

Let $S_{p}$ denote $\sum_{n=p}^{\infty}\left|F_{2 n+j}-F_{2 n-2+j}\right|$ and let $k_{2 p-1+j}$ denote the number

$$
\frac{S_{p+1}}{S_{p}} \cdot \frac{\left|B_{2 p+j}\right|}{\left|a_{2 p-1+j} B_{2 p-2+j}\right|}
$$

Now, $k_{2 p-1+j}>0$,

and

$$
k_{2 p-1+j}\left|a_{2 p-1+j} B_{2 p-2+j}\right|=\frac{S_{p+1}}{S_{p}}\left|B_{2 p+j}\right|,
$$

$$
\begin{aligned}
\left|B_{2 p+j}\right|-k_{2 p-1+j}\left|a_{2 p-1+j} B_{2 p-2+j}\right| & =\left|B_{2 p+j}\right|\left(1-\frac{S_{p+1}}{S_{p}}\right) \\
& =\left|B_{2 p+j}\right| \frac{\left|F_{2 p+j}-F_{2 p-2+j}\right|}{S_{p}} .
\end{aligned}
$$

In particular,

$$
\left|B_{2+j}\right|-k_{1+j}\left|a_{1+j} B_{j}\right|=\frac{\left|B_{2+j}\right|}{S_{1}}\left|F_{2+j}-F_{j}\right|=\frac{\left|a_{j} b_{2+j}\right|}{S_{1}\left|B_{j}\right|}
$$

and thus if $M_{j}=\left|a_{j}\right| / S_{1}\left|B_{j}\right|$, then

$$
\frac{\left|B_{2+j}\right|-k_{1+j}\left|a_{1+j} B_{j}\right|}{\left|b_{2+j}\right|}=M_{j}>0 .
$$

Also, 


$$
\begin{aligned}
& \frac{\left|B_{2 p+2+j}\right|-k_{2 p+1+j}\left|a_{2 p+1+j} B_{2 p+j}\right|}{\left|b_{2 p+2+j}\right|} \\
& =\frac{\left|a_{2 p+j}\right|}{k_{2 p-1+j}} \frac{\left|B_{2 p+j}\right|-k_{2 p-1+j}\left|a_{2 p-1+j} B_{2 p-2+j}\right|}{\left|b_{2 p+j}\right|} ;
\end{aligned}
$$

thus, statement (1) is true with equality holding throughout. This completes the proof of Theorem A.

3. Some convergence theorems proved by use of Theorem $\mathbf{A}$.

ExAmple 3.1. If $0<g_{p}<1$ and $\left|a_{p}\right| \leqq\left(1-g_{p}\right) g_{p+1}$, then the even and odd parts of the continued fraction $f(a)$ converge absolutely and $f(a)$ converges.

Proof. The " $k$-inequalities" (2.1) hold if $M_{0}=g_{1}, M_{1}=g_{1}\left|a_{1}\right| / 1-g_{1}$, and $k_{p}=\left(1-g_{p+1}\right) / g_{p+1}$; thus, by Theorem $\mathrm{A}$, the even and odd parts of $f(a)$ converge absolutely. Since $a \in V$, then by Theorem LW, $f(a)$ converges. Moreover, from inequality (2.2), $\left|\lim _{n \rightarrow \infty} f_{n}\right| \leqq 1 / g_{1}$.

The following is a generalization of "The Parabola Theorem" of Scott and Wall [3] (and reduces to "The Parabola Theorem" if $t=1 / 2)$.

EXAMPLE 3.2. If $0<t<1,\left|a_{2 p-1}\right|-\operatorname{Re} a_{2 p-1} \leqq t$, and $\left|a_{2 p}\right|-\operatorname{Re} a_{2 p}$ $\leqq 1-t$, then the even and odd parts of $f(a)$ converge absolutely and $f(a)$ converges only if $a \in V$.

PROOF. The " $k$-inequalities" (2.1) hold if $M_{0}=1-t, M_{1}=\left|a_{1}\right|$, and $k_{p}=1$; thus, by Theorem A, the even and odd parts of $f(a)$ converge absolutely and hence Theorem LW applies. If $f(a)$ converges to $L$, then from inequality (2.2), $|L| \leqq 1 /(1-t)$ and $|L| \leqq 2$.

4. Concerning the impossibility of a certain extension of "The Parabola Theorem." The conjecture of Wall, described in the introduction of this paper, will now be considered. For this discussion, it is convenient to introduce the following definition.

Definition 4.1. A number sequence $g$ has property $\mathrm{P}$ only if (i) $0<g_{p}<1$ and (ii) if $a$ is a complex number sequence such that $a \in V$ and $\left|a_{p}\right|-\operatorname{Re} a_{p} \leqq 2\left(1-g_{p}\right) g_{p+1}$, then the continued fraction $f(a)$ converges.

Note 4.1 . The number sequence $1 / 2,1 / 2,1 / 2, \cdots$ has property $\mathrm{P}$; this statement, in fact, is precisely "The Parabola Theorem" of Scott and Wall [3].

The following theorem gives a necessary condition for a number sequence, each term of which is between 0 and 1 , to have property $P$. 
THEOREM B. Suppose that $g$ is a number sequence such that $0<g_{2}<1$ and $c_{p}=\left(1-g_{p}\right) g_{p+1}$. In order for the sequence $g$ to have property $\mathrm{P}$, it is necessary that each of the sequences $\left\{c_{2 p+1} /\left(1-c_{2 p+1}\right)\right\}_{p=1}^{\infty}$ and $\left\{c_{2 p} /\left(1-c_{2 p}\right)\right\}_{p=1}^{\infty}$ have property V.

PROOF. If $\left\{f_{p}\right\}_{p=1}^{\infty}$ is the sequence of approximants of the continued fraction $f(a)$, then the continued fraction

$$
1+\frac{d_{1}}{1}+\frac{d_{2} d_{3}}{1}+\frac{d_{4} d_{5}}{1}+\ldots
$$

where

$$
d_{2 p-1}=\frac{-a_{2 p-1}}{1+a_{2 p-1}+a_{2 p}}
$$

and

$$
d_{2 p}=\frac{a_{2 p}}{1+a_{2 p-1}+a_{2 p}}
$$

has the sequence of approximants $f_{1}, f_{3}, f_{5}, \ldots$. Suppose that $a_{2 p-1}=-c_{2 p-1}$ and $a_{2 p}=1-c_{2 p-1}$. Note first that $-1<a_{2 p-1}<0<a_{2 p}$ $<1$; thus $a \in V$. Also, $\left|a_{p}\right|-\operatorname{Re} a_{p} \leqq 2\left(1-g_{p}\right) g_{p+1}$. It is easily verified that $d_{2 p} d_{2 p+1}=4^{-1} c_{2 p+1} /\left(1-c_{2 p+1}\right)$. If the sequence $\left\{c_{2 p+1} /\left(1-c_{2 p+1}\right)\right\}_{p=1}^{\infty}$ does not have property $V$, then the sequence $\left\{4^{-1} c_{2 p+1} /\left(1-c_{2 p+1}\right\}_{p=1}^{\infty}\right.$ does not have property $\mathrm{V}$, the continued fraction (4.1) diverges, and thus the continued fraction $f(a)$ diverges. A similar discussion, using the even part of $f(a)$, yields the necessity that the sequence $\left\{c_{2 p} /\left(1-c_{2 p}\right)\right\}_{p=1}^{\infty}$ have property $\mathrm{V}$.

EXAMPLE. Suppose that $g_{2 p-1}=1 / 1+2^{2 p}, g_{2 p}=1-1 / 2+2^{2 p}, a_{2 p-1}$ $=-\left(1-g_{2 p-1}\right) g_{2 p}$, and $a_{2 p}=1-\left(1-g_{2 p-1}\right) g_{2 p}$. Then $0<g_{p}<1, a \in V$, $\left|a_{p}\right|-\operatorname{Re} a_{p} \leqq 2\left(1-g_{p}\right) g_{p+1}$, and the continued fraction (4.1) becomes (4.2) $1+f(\alpha)$, where $\alpha_{p}=2^{2 p-1}$. Now if $b_{1}=1$, and $b_{p+1}=1 / b_{p} \alpha_{p}$, then $\sum_{p=1}^{\infty}\left|b_{p}\right|=2$; i.e., the sequence $\alpha$ does not have property V. Consequently, the continued fractions (4.2) and $f(a)$ diverge. In fact, for this example, if $\left\{f_{p}\right\}_{p=0}^{\infty}$ is the sequence of approximants of the continued fraction $f(a)$, then the sequences $\left\{f_{4 p+1}\right\}$ and $\left\{f_{4 p+3}\right\}$ converge, but not to the same limit, and the sequences $\left\{f_{4 p}\right\}$ and $\left\{f_{4 p+2}\right\}$ converge, but not to the same limit. This example settles in the negative the previously described conjecture of Wall. Incidentally, for this example, it can be shown that $\lim _{p \rightarrow \infty} f_{4 p+1}=\lim _{p \rightarrow \infty} f_{4 p}$ and $\lim _{p \rightarrow \infty} f_{4 p+3}$ $=\lim _{p \rightarrow \infty} f_{4 p+2}$. 


\section{BIBLIOGRAPHY}

1. R. E. Lane, Absolute convergence of continued fractions, Proc. Amer. Math. Soc. vol. 3 (1952) pp. 904-913.

2. R. E. Lane and H. S. Wall, Continued fractions with absolutely convergent even and odd parts, Trans. Amer. Math. Soc. vol. 67 (1949) pp. 368-380.

3. W. T. Scott and H. S. Wall, A convergence theorem for continued fractions, Trans. Amer. Math. Soc. vol. 47 (1940) pp. 155-172.

4. H. S. Wall, Analytic theory of continued fractions, New York, D. Van Nostrand Company, Inc., 1948.

The University OF TeXas AND

UNIVERSITY OF HOUSTON

\section{QUASI-BARRELLED LOCALLY CONVEX SPACES}

\section{MARK MAHOWALD AND GERALD GOULD}

1. Introduction and preliminary definitions. The main object of this paper is to answer some problems posed by Dieudonné in his paper Denumerability conditions in locally convex vector spaces [2]. His two main results are as follows:

PROPOSITION 1. If $E$ is a barrelled space on which there is a countable fundamental system of convex compact subsets, [Definition 1.2] then it is the strong dual of a Fréchet-Montel Space.

PROPOSITION 2. If $E$ is either bornological or barrelled, and if there is a countable fundamental system of compact subsets, then $E$ is dense in the strong dual of a Frêchet-Montel Space.

Two questions raised by Dieudonné in connection with these results are:

(a) If $E$ is either bornological or barrelled then it is certainly quasibarrelled [1, Chapter 3, §2, Example 12]. Can one substitute this weaker condition on $E$ in Proposition 2?

(b) Is there is an example of a quasi-barrelled space which is neither barrelled nor bornological?

We shall show that the answer to (a) is "Yes," and that the answer to (b) is also "Yes," so that the generalization is in fact a real one.

A knowledge of the main results and notation of [1] is assumed. References to results occurring in the exercises there will however be given. These include in particular all the definitions and theorems

Presented to the Society January 29, 1960; received by the editors January 7, 1960. 